\title{
Bisymmetric functionals revisited or a converse of the Fubini theorem
}

\section{Maciej SABlikiD}

Dedicated to Professor János Aczél on his 95th Birthday.

\begin{abstract}
We observe that bisymmetry is in fact the assertion of the Fubini theorem and we describe the form of general bisymmetric operations on some function spaces.
\end{abstract}

Mathematics Subject Classification. 39B52, 43A07, 47A67.

Keywords. Functional equations, Bisymmetry, Fubini theorem, Representation of bisymmetric functionals.

\section{Introduction}

Let us observe that the well known arithmetic mean may be treated as a functional on the space of all functions from $\mathbb{Z}_{2}$ to $\mathbb{R}$. In fact, if $x: \mathbb{Z}_{2} \longrightarrow \mathbb{R}$ is an arbitrary function then the arithmetic mean $M(x)$ is given by

$$
M(x)=\frac{x(0)+x(1)}{2}, x: \mathbb{Z}_{2} \longrightarrow \mathbb{R} .
$$

It is rather easy to observe that $M$ satisfies the following conditions:

(a) $M$ is linear,

(b) $M(x) \leq \sup x(G)(\Longleftrightarrow M(x) \geq \inf x(G))$,

(c) $M$ is translation invariant (i.e. $M\left(x_{a}\right)=M(x)$ for every $a \in G$, where $\left.x_{a}(t)=x(t a), t \in G\right)$,

where $G$ is an arbitrary group, in our case $G=\mathbb{Z}_{2}$. Slightly more generally, if we fix arbitrary $n \geq 2$, then a functional $M$ satisfying (a), (b) and (c) and defined on $\mathcal{B}\left(\mathbb{Z}_{n} ; \mathbb{R}\right)$ (i.e. the family of all bounded functions mapping $\mathbb{Z}_{n}$ into $\mathbb{R}$ - in fact, all the mappings from $\mathbb{Z}_{n}$ into $\mathbb{R}$ ) has to be 
(d) continuous;

(e) reflexive, i. e.

$$
M(c \mathbf{1})=c
$$

for every $c \in \mathbb{R}$, here $\mathbf{1}: G \longrightarrow \mathbb{R}$ is defined by $\mathbf{1}(t)=1, t \in G$;

(f) monotonic, i. e.

$$
x \geq 0 \Longrightarrow M(x) \geq 0 .
$$

Now, from (a) we infer that

$$
M(x)=\sum_{k=0}^{n-1} w_{k} x(k),
$$

and (1.1) implies

$$
\sum_{k=0}^{n-1} w_{k}=1
$$

Moreover, because of (f) ((1.2), which is a consequence of (b)) we obtain

$$
\bigwedge_{k \in\{0, \ldots, n-1\}} w_{k} \geq 0
$$

Further, because of (c) we get

$$
\bigwedge_{k \in\{0, \ldots, n-1\}} \bigwedge_{j \in\{0, \ldots, n-1\}} w_{k+j}=w_{k},
$$

in other words

$$
\bigwedge_{k \in\{0, \ldots, n-1\}} w_{k}=\frac{1}{n} .
$$

Hence, if $M$ satisfies (a), (f) and (c) then it has to be the arithmetic mean.

Therefore, we have to develop some other instruments to investigate means on finite groups. It turns out that linearity is in a sense too much - it implies the form (1.3). We know several means that are not linear, but bisymmetric, i.e. satisfy the functional equation

$$
M[M(x, y), M(u, v)]=M[M(x, u), M(y, v)] .
$$

\subsection{History}

In the 1940's Aczél [1, p. 281] proved the following theorem on the form of bisymmetric means. 
Theorem 1.1. The quasi-arithmetic mean

$$
M(x, y)=\varphi^{-1}\left(\frac{\varphi(x)+\varphi(y)}{2}\right)
$$

is the general continuous, reducible on both sides, ${ }^{1}$ real solution of

$$
\begin{aligned}
& M\left[M\left(x_{00}, x_{01}\right), M\left(x_{10}, x_{11}\right)\right] \\
& \quad=M\left[M\left(x_{00}, x_{10}\right), M\left(x_{01}, x_{11}\right)\right]
\end{aligned}
$$

under the additional conditions $x, y, M(x, y) \in[a, b]$,

$$
\begin{aligned}
& M(x, x)=x \quad \text { for all } x \in[a, b], \\
& M(x, y)=M(y, x) \quad \text { for all } x, y \in[a, b] .
\end{aligned}
$$

(Without symmetry $M$ is given by

$$
M(x, y)=\varphi^{-1}((1-q) \varphi(x)+q \varphi(y))
$$

where $q \in(0,1)$.)

Remark 1.1. Without reflexivity and symmetry $M$ is given by

$$
M(x, y)=\varphi^{-1}((\alpha \varphi(x)+\beta \varphi(y)+\gamma)
$$

for some $\alpha>0, \beta>0$ and $\gamma \in \mathbb{R}$ such that

$$
u, v \in[a, b] \Longrightarrow \alpha u+\beta v+\gamma \in[a, b] .
$$

(Volkmann [12], Maksa [9]).

Remark 1.2. It is noteworthy that the formula expressing bisymmetry is actually a form of the equality appearing in the celebrated Fubini theorem on iterated integrals.

A more general result was obtained by Münnich et al. [11] (Theorem 2).

Theorem 1.2. Let $I$ be a real open interval, bounded or not, and let $F: I^{n} \rightarrow I$ ( $n \geq 2$ fixed) be strictly monotone increasing in each of its arguments, continuous, and satisfy

$$
\begin{aligned}
& F\left(F\left(x_{00}, \ldots, x_{0 n-1}\right), \ldots, F\left(x_{n-10}, \ldots, x_{n-1 n-1}\right)\right) \\
& \quad=F\left(F\left(x_{00}, \ldots, x_{n-10}\right), \ldots, F\left(x_{0 n-1}, \ldots, x_{n-1 n-1}\right)\right)
\end{aligned}
$$

and

$$
F(x, \ldots, x)=x, \quad x \in I .
$$

Then there are strictly monotone increasing, continuous function $\varphi: I \rightarrow \mathbb{R}$ and constants $\alpha_{0}, \ldots, \alpha_{n-1} \in(0,1)$ such that

$$
\sum_{i=0}^{n-1} \alpha_{i}=1
$$

\footnotetext{
${ }^{1} M:[a, b]^{2} \rightarrow[a, b]$ is reducible on both sides iff for every $x, y, z \in[a, b] M(x, y)=M(x, z)$ implies $y=z$ and $M(x, y)=M(z, y)$ implies $x=z$.
} 
and

$$
F\left(x_{0}, \ldots, x_{n-1}\right)=\varphi^{-1}\left(\sum_{i=0}^{n-1} \alpha_{i} \varphi\left(x_{i}\right)\right) .
$$

There is also another result in this spirit. Let $A, B \in \mathbb{R}, A<B$ and denote by $\mathcal{D}(A, B)$ the family of all distribution functions $F: \mathbb{R} \longrightarrow[0,1]$ such that

- $F$ is constant in stretches and has only a finite number of discontinuities;

- $F(x)=\frac{1}{2}[F(x+)-F(x-)],^{2} \quad x \in \mathbb{R}$;

- $F(x)=0, x \leq A$ and $F(x)=1, x \geq B$.

Note that for every $\xi \in[A, B]$ the function $E_{\xi}$ given by $E_{\xi}(x)=\frac{1}{2}(1+\operatorname{sgn}(x-$ $\xi)$ ) belongs to $\mathcal{D}(A, B)$. The following was proved by de Finetti [5] (cf. Hardy et al. [7, p. 158]).

Theorem 1.3. Suppose that $M: \mathcal{D}(A, B) \longrightarrow \mathbb{R}$ satisfies

(a) $M\left(E_{\xi}\right)=\xi, \xi \in[A, B]$;

(b) if $F_{1}, F_{2} \in \mathcal{D}(A, B), F_{1} \geq F_{2}$, and $F_{1}(x)>F_{2}(x)$ for some $x \in \mathbb{R}$, then $M\left(F_{1}\right)<M\left(F_{2}\right)$

(c) if $F, F^{*}, G \in \mathcal{D}(A, B)$ and $M(F)=M\left(F^{*}\right)$, then

$$
M(t F+(1-t) G)=M\left(t F^{*}+(1-t) G\right)
$$

for $t \in(0,1)$.

Then there is a function $\phi$, continuous and strictly increasing in $[A, B]$, for which

$$
M(F)=\phi^{-1}\left(\int_{-\infty}^{\infty} \phi(x) d F(x)\right) .
$$

Conversely, if $M$ is defined by $\sharp)$, for a $\phi$ with the properties stated, then it satisfies $(a),(b)$ and $(c)$, so that these conditions are necessary and sufficient for the representation of $M$ in the form $(\sharp)$.

\subsection{Consistent aggregation}

One of the problems in which bisymmetric operations naturally arise is the so called consistent aggregation, appearing in the procedure of aggregating outputs of different producers who, with different production functions, make several goods. In this connection the following equation comes up (cf. e.g. [2])

$$
\begin{aligned}
& G\left(F_{1}\left(x_{0,0}, \ldots, x_{n-1,0}\right), \ldots, F_{m}\left(x_{0, m-1}, \ldots, x_{n-1, m-1}\right)\right) \\
& \quad=F\left(G_{1}\left(x_{0,0}, \ldots, x_{0, m-1}\right), \ldots, G_{n}\left(x_{n-1,0}, \ldots, x_{n-1, m-1}\right)\right)
\end{aligned}
$$

${ }^{2} F(x \pm)=\lim _{y \rightarrow x \pm} F(y)$. 
or

$$
\begin{aligned}
& G(F(1, x(\cdot, 0)), \ldots, F(m, x(\cdot, m-1))) \\
& \quad=F(G(1, x(0, \cdot)), \ldots, G(n, x(n-1, \cdot))) .
\end{aligned}
$$

Equation (1.6) was solved by Maksa $[9,10]$.

Example 1. Put for every $y: A=\{0, \ldots, n-1\} \longrightarrow \mathbb{R}$

$$
F(y)=F(1, y)=F(2, y)=\cdots=F(m, y)=\sum_{s=0}^{n-1} y(s)=\int_{A} y(s) d s .
$$

Similarly, let $B=\{0, \ldots, m-1\}$. For every $z: B \longrightarrow \mathbb{R}$, let us put

$$
G(z)=G(1, z)=\cdots=G(n, z)=\sum_{t=0}^{m-1} z(t)=\int_{B} z(t) d t .
$$

Then for every $x: A \times B \longrightarrow \mathbb{R},(1.6)$ becomes

$$
\begin{aligned}
& \sum_{t=0}^{m-1}\left(\sum_{s=0}^{n-1} x(s, t)\right)=\int_{B}\left(\int_{A} x(s, t) d s\right) d t \\
& \quad=\int_{A}\left(\int_{B} x(s, t) d t\right) d s=\sum_{s=0}^{n-1}\left(\sum_{t=0}^{m-1} x(s, t)\right) .
\end{aligned}
$$

Now, it is clearly seen that "consistent aggregation" is in fact the "Fubini theorem".

Finally, let us mention the quite recent papers by Leonetti et al. [8] and Głazowska with the former authors [6] where a question of the commutativity of integral means was considered. The results are also in the spirit of the Fubini theorem. We shall use one of their theorems to prove our main result.

In the sequel we will formulate a proposition which characterizes means satisfying bisymmetry or, as we have tried to convince the reader, the assertion of the Fubini theorem. We prove Proposition 3.1 for families of finite valued functions and then extend its result to the case of some function spaces, in which finite valued functions form a dense subset (cf. Corollaries 3.1, 3.2 and 3.3). In order to do this however, we need to introduce some notations and definitions.

\section{Notations and definitions}

Consider $(\Omega, \mathcal{A}, \mu)$, where $\Omega \neq \emptyset, \mathcal{A} \subset 2^{\Omega}$ is an algebra of sets, and $\mu: \mathcal{A} \rightarrow$ $[0, \infty]$ is an additive and nontrivial set function. Further, let $\mathcal{M}(\Omega)=\{f: \Omega \rightarrow$ $\mathbb{R}: f$ is $\mathcal{A}$-measurable $\}$ and $\mathcal{M}_{+}(\Omega)=\left\{f: \Omega \rightarrow \mathbb{R}_{+}: f\right.$ is $\mathcal{A}$-measurable $\}$. 
Introduce a relation $\leq$ in $\mathcal{M}(\Omega)\left(\mathcal{M}_{+}(\Omega)\right)$ in the following way. If $f, g \in$ $\mathcal{M}(\Omega)\left(\mathcal{M}_{+}(\Omega)\right)$ then

$$
f \leq g \Longleftrightarrow \mu(\{\omega \in \Omega: f(\omega)>g(\omega)\})=0 .
$$

Actually, $\leq$ is a preorder in $\mathcal{M}(\Omega)\left(\mathcal{M}_{+}(\Omega)\right)$, since $f \leq g$ and $g \leq f$ imply that $f=g \mu$-almost everywhere. Also, we say that for $f, g \in \mathcal{M}(\Omega)\left(\mathcal{M}_{+}(\Omega)\right)$

$$
f<g \Longleftrightarrow f \leq g \wedge f \neq g \mu \text {-a.e., }
$$

in other words

$$
f<g \Longleftrightarrow f \leq g \wedge \mu(\{\omega \in \Omega: f(\omega)<g(\omega)\})>0 .
$$

For each $n \in \mathbb{N}$ we call a sequence of sets $\left(A_{0}, \ldots, A_{n-1}\right) \subset \mathcal{A}^{n}$ satisfying

$$
\left(\bigcup_{k=0}^{n-1} A_{k}=\Omega\right) \wedge\left(k \neq j \Longrightarrow A_{k} \cap A_{j}=\emptyset\right)
$$

a partition of $\Omega$. The family of all partitions of $\Omega$ will be denoted by $\mathbf{P}(\Omega)$ in the sequel. Observe that

$$
\begin{aligned}
\mathbf{F}(\Omega)= & \{f \in \mathcal{M}(\Omega): f(\Omega) \text { is finite }\}=\left\{\sum_{k=0}^{n-1} x_{k} \mathbf{1}_{A_{k}}: n \in \mathbb{N},\right. \\
& \left.\left(A_{0}, \ldots, A_{n-1}\right) \in \mathbf{P}(\Omega), x_{k} \in \mathbb{R}, k \in\{0, \ldots, n-1\}\right\}
\end{aligned}
$$

and analogously

$$
\begin{aligned}
\mathbf{F}_{+}(\Omega):= & \left\{f \in \mathcal{M}_{+}(\Omega): f(\Omega) \text { is finite }\right\}=\left\{\sum_{k=0}^{n-1} x_{k} \mathbf{1}_{A_{k}}: n \in \mathbb{N},\right. \\
& \left.\left(A_{0}, \ldots, A_{n-1}\right) \in \mathbf{P}(\Omega), x_{k} \geq 0, k \in\{0, \ldots, n-1\}\right\} .
\end{aligned}
$$

We will also consider the following families of functions $x: \Omega \times \Omega \longrightarrow \mathbb{R}$ :

$$
\begin{aligned}
& \mathbf{F}(\Omega \times \Omega):=\left\{\sum_{i=0}^{n-1} \sum_{j=0}^{m-1} x_{i j} \mathbf{1}_{A_{i} \times B_{j}}: m, n \in \mathbb{N},\left(A_{0}, \ldots, A_{n-1}\right)\right. \\
& \left.\left(B_{0}, \ldots, B_{m-1}\right) \in \mathbf{P}(\Omega), x_{i j} \in \mathbb{R}, i \in\{0, \ldots, n-1\}, j \in\{0, \ldots, m-1\}\right\}
\end{aligned}
$$


and

$$
\begin{aligned}
& \mathbf{F}_{+}(\Omega \times \Omega):=\left\{\sum_{i=0}^{n-1} \sum_{j=0}^{m-1} x_{i j} \mathbf{1}_{A_{i} \times B_{j}}: m, n \in \mathbb{N},\left(A_{0}, \ldots, A_{n-1}\right),\right. \\
& \left.\left(B_{0}, \ldots, B_{m-1}\right) \in \mathbf{P}(\Omega), x_{i j} \geq 0, i \in\{0, \ldots, n-1\}, j \in\{0, \ldots, m-1\}\right\} .
\end{aligned}
$$

Our aim is to study functionals defined on families of measurable functions, containing the finite valued ones. First, let us define the properties of functionals in question.

Let $\mathcal{F} \subset \mathcal{M}(\Omega)\left(\mathcal{M}_{+}(\Omega)\right)$ be such that $\mathbf{F}(\Omega) \subset \mathcal{F}\left(\mathbf{F}_{+}(\Omega) \subset \mathcal{F}\right)$. We say that a functional $M: \mathcal{F} \rightarrow \mathbb{R}$ makes no distinction between functions equal $\underline{\mu-\text { a.e. }}$ iff

$$
f=g \mu \text {-almost everywhere } \Longrightarrow M(f)=M(g) .
$$

We say that $M$ is reflexive, iff

$$
M(c \mathbf{1})=c \text { for every } c \in \mathbb{R}\left(\mathbb{R}_{+}\right) .
$$

Moreover, $M$ is $\mu$-strictly increasing, iff

$$
f<g \Longrightarrow M(f)<M(g)
$$

for every $f, g \in \mathcal{F}$ (cf. (2.2) for the definition of $f<g$ ).

Remark 2.1. Fix a partition $\left(A_{0}, \ldots, A_{n-1}\right) \in \mathbf{P}(\Omega)$. Take two functions from $\mathbf{F}(\Omega)\left(\mathbf{F}_{+}(\Omega)\right)$ corresponding to the partition, say

$$
x=\sum_{k=0}^{n-1} x_{k} \mathbf{1}_{A_{k}}
$$

and $y=\sum_{k=0}^{n-1} y_{k} \mathbf{1}_{A_{k}}$. Assume that $x<y$. In view of (2.2) we get that $x_{k} \leq y_{k}$, for each $k \in\{0, \ldots, n-1\}$, unless $\mu\left(A_{k}\right)=0$. For some $k_{0} \in\{0, \ldots, n-1\}$ we have to get $x_{k_{0}}<y_{k_{0}}$, and the latter has to occur together with $\mu\left(A_{k_{0}}\right)>0$.

Remark 2.2. As usual, we will not distinguish functions that are equal $\mu$ almost everywhere, cf. the assumption (2.6). Therefore in the definition of $\mathbf{F}(\Omega)$ and $\mathbf{F}_{+}(\Omega)$, we shall restrict ourselves to the case of such partitions $\left(A_{0}, \ldots, A_{n-1}\right)$ that for each $k \in\{0, \ldots, n-1\}$ we have $\mu\left(A_{k}\right)>0$. More exactly, from the equivalence class of functions equal $\mu$-a.e. we shall pick those which correspond to partitions not including nullsets. It is always possible, just by joining the nullsets to the sets of positive measure and taking the corresponding value. In the sequel we will deal also with some partitions with sets of measure zero as components, this will be the case for instance if we take a partition which results as the intersection of two partitions. More exactly, if $\Pi=\left(A_{0}, \ldots, A_{n-1}\right)$ and $\Pi^{\prime}=\left(B_{0}, \ldots, B_{m-1}\right)$ are two partitions of $\Omega$, then $\Pi \wedge \Pi^{\prime}:=\left(A_{0} \cap B_{0}, \ldots, A_{i} \cap B_{j}, \ldots, A_{n-1} \cap B_{m-1}\right) \in \mathbf{P}(\Omega)$ as well but some of 
the sets $A_{i} \cap B_{j}$ might be $\mu$-null sets. The function $y^{\prime}=\sum_{i=0}^{n-1} \sum_{j=0}^{m-1} y_{i j} \mathbf{1}_{A_{i} \cap B_{j}}$ will be replaced by an equivalent one $y=\sum_{(i, j) \in L(m, n)} y_{i j} \mathbf{1}_{C_{i j}}$ where $L(m, n)$ denotes the set of all pairs $(i, j) \in\{0, \ldots, m-1\} \times\{0, \ldots, n-1\}$ such that $\mu\left(A_{i} \cap B_{j}\right)>0$, and $C_{i j}=A_{i} \cap B_{j}$ except for one pair $\left(i_{0}, j_{0}\right) \in L(m, n)$ for which $C_{i_{0} j_{0}}=A_{i_{0}} \cap B_{j_{0}} \cup \bigcup_{(r, s) \notin L(m, n)} A_{r} \cap B_{s}$. Then the function $y$ corresponds to a partition containing sets with positive measure only. We will use the partition $\Pi \wedge \Pi^{\prime}$ in the proof of Proposition 3.1.

Let us introduce a new definition.

Definition 2.1. Let $\mathcal{F} \subset \mathcal{M}(\Omega)\left(\mathcal{M}_{+}(\Omega)\right)$ be such that $\mathbf{F}(\Omega) \subset \mathcal{F}\left(\mathbf{F}_{+}(\Omega) \subset\right.$ $\mathcal{F})$ and let $M: \mathcal{F} \rightarrow \mathbb{R}$. If $x: \Omega \times \Omega \rightarrow \mathbb{R}$ is such that $x(\cdot, t) \in \mathcal{F}$ for every $t \in \Omega$ then we put

$$
M_{1}(x):=" \Omega \ni t \rightarrow M(x(\cdot, t)) \in \mathbb{R} ",
$$

and if $x(s, \cdot) \in \mathcal{F}$ for every $s \in \Omega$ then we put

$$
M_{2}(x):=" \Omega \ni s \rightarrow M(x(s, \cdot)) \in \mathbb{R} " .
$$

We say that a function $x: \Omega \times \Omega \rightarrow \mathbb{R}$ is admissible if $x(\cdot, t) \in \mathcal{F}$ for every $t \in \Omega$ and $x(s, \cdot) \in \mathcal{F}$ for every $s \in \Omega$. We show the following.

Lemma 2.1. Let $x \in \mathbf{F}(\Omega \times \Omega)\left(\mathbf{F}_{+}(\Omega \times \Omega)\right)$. Then $x$ is admissible and

$$
M_{1}(x) \in \mathbf{F}(\Omega)\left(\mathbf{F}_{+}(\Omega)\right)
$$

and

$$
M_{2}(x) \in \mathbf{F}(\Omega)\left(\mathbf{F}_{+}(\Omega)\right) .
$$

Proof. Suppose that

$$
\begin{aligned}
x(s, t) & =\sum_{i=0}^{n-1} \sum_{j=0}^{m-1} x_{i j} \mathbf{1}_{A_{i} \times B_{j}}(s, t) \\
& =\sum_{i=0}^{n-1} \sum_{j=0}^{m-1} x_{i, j} \mathbf{1}_{A_{i}}(s) \mathbf{1}_{B_{j}}(t),
\end{aligned}
$$

for all $s, t \in \Omega$, where $\left(A_{0}, \ldots, A_{n-1}\right)$ and $\left(B_{0}, \ldots, B_{m-1}\right)$ are two partitions of $\Omega$ (cf. (2.3)). Then $x(\cdot, t) \in \mathbf{F}(\Omega)\left(\mathbf{F}_{+}(\Omega)\right)$ for every $t \in \Omega$ and (cf. (2.9))

$$
M_{1}(x)(t)=M\left(\sum_{i=0}^{n-1} x_{i, j} \mathbf{1}_{A_{i}}\right)
$$

for all $t \in B_{j}, j \in\{1, \ldots, m-1\}$. We can write it in the form

$$
M_{1}(x)=\sum_{j=0}^{m-1} M\left(\sum_{i=0}^{n-1} x_{i, j} \mathbf{1}_{A_{i}}\right) \mathbf{1}_{B_{j}},
$$


which proves our Lemma. Similarly we prove that (cf. (2.10))

$$
M_{2}(x)=\sum_{i=0}^{n-1} M\left(\sum_{j=0}^{m-1} x_{i, j} \mathbf{1}_{B_{j}}\right) \mathbf{1}_{A_{i}},
$$

and so $M_{2}(x) \in \mathbf{F}(\Omega)$.

Example 2. Suppose that $\Omega=\left\{\omega_{1}, \ldots, \omega_{r}\right\}$ for some $r \in \mathbb{N}$, let $\mathcal{A}=2^{\Omega}, \mu\left(\omega_{s}\right)$ $=\frac{1}{r}$ for every $s \in\{1, \ldots, r\}$ and consider a function $x: \Omega \times \Omega \longrightarrow \mathbb{R}$, defined by

$$
x(s, t)=\sum_{i, j} x_{i, j} \mathbf{1}_{\left\{\left(\omega_{i}, \omega_{j}\right)\right\}}(s, t)=: x_{s, t}
$$

for every $(s, t) \in \Omega \times \Omega$. Obviously, $x \in \mathbf{F}(\Omega \times \Omega)$. Let $M: \mathbf{F}(\Omega) \longrightarrow \mathbb{R}$ be any function which is reflexive and strictly increasing, for instance suppose that

$$
M(z)=M\left(\sum_{s=1}^{r} z_{s} \mathbf{1}_{\left\{\omega_{s}\right\}}\right)=\sum_{s=1}^{r} \lambda_{s} z_{s},
$$

for every $z=\sum_{s=1}^{r} z_{s} \mathbf{1}_{\left\{\omega_{s}\right\}}$ where $\left(\lambda_{1}, \ldots, \lambda_{r}\right)$ is a fixed vector such that $\lambda_{s}>0, s \in\{1, \ldots, r\}$ and $\sum_{s=1}^{r} \lambda_{s}=1$. Then we have

$$
M_{1}(x)(t)=\sum_{s=1}^{r} \lambda_{s} x_{s, t} \in \mathbb{R}, t \in\{1, \ldots, r\}
$$

and

$$
M_{2}(x)(s)=\sum_{t=1}^{r} \lambda_{t} x_{s, t} \in \mathbb{R}, s \in\{1, \ldots, r\} .
$$

Remark 2.3. Let us observe the following. Fix $m \geq 1$ and $n \geq 1$, positive integers. Further, fix partitions $\left(A_{0}, \ldots, A_{n-1}\right)$ and $\left(B_{1}, \ldots, B_{m-1}\right) \in \mathbf{P}(\Omega)$. We assume that for every $i \in\{1, \ldots, n-1\}$ and for every $j \in\{1, \ldots, m-1\}$ we have

$$
\mu\left(A_{i}\right) \mu\left(B_{j}\right)>0 .
$$

Let us choose arbitrary $x_{i j} \in \mathbb{R}, i \in\{0, \ldots, n-1\}, j \in\{0, \ldots, m-1\}$. Consider a function $x \in \mathbf{F}(\Omega \times \Omega)\left(\mathbf{F}_{+}(\Omega \times \Omega)\right)$, defined by (2.11). We get by (2.12)) and (2.13) that $M_{k}(x) \in \mathbf{F}(\Omega)\left(\mathbf{F}_{+}(\Omega), k \in\{1,2\}\right.$. Finally, assume that

$$
M\left(M_{1}(x)\right)=M\left(M_{2}(x)\right) .
$$


Define $F: \mathbb{R}^{n} \longrightarrow \mathbb{R}$ and $G: \mathbb{R}^{m} \longrightarrow \mathbb{R}\left(F: \mathbb{R}_{+}^{n} \longrightarrow \mathbb{R}, G: \mathbb{R}_{+}^{m} \longrightarrow \mathbb{R}\right)$ by

$$
\begin{aligned}
& F\left(x_{0}, \ldots, x_{n-1}\right)=M\left(\sum_{i=0}^{n-1} x_{i} \mathbf{1}_{A_{i}}\right),\left(x_{0}, \ldots, x_{n-1}\right) \in \mathbb{R}^{n}\left(\mathbb{R}_{+}^{n}\right) ; \\
& G\left(y_{0}, \ldots, y_{m-1}\right)=M\left(\sum_{j=0}^{m-1} y_{j} \mathbf{1}_{B_{j}}\right),\left(y_{0}, \ldots, y_{m-1}\right) \in \mathbb{R}^{m}\left(\mathbb{R}_{+}^{m}\right) .
\end{aligned}
$$

In view of (2.15) we get

$$
\begin{aligned}
F(G & \left.\left(x_{00}, \ldots, x_{0 m-1}\right), \ldots, G\left(x_{n-10}, \ldots, x_{n-1 m-1}\right)\right) \\
& =F\left(M\left(\sum_{j=0}^{m-1} x_{0 j} \mathbf{1}_{B_{j}}\right), \ldots, M\left(\sum_{j=0}^{m-1} x_{n-1 j} \mathbf{1}_{B_{j}}\right)\right) \\
& =M\left(\sum_{i=0}^{n-1} M\left(\sum_{j=0}^{m-1} x_{i j} \mathbf{1}_{B_{j}}\right) \mathbf{1}_{A_{i}}\right) \\
& =M\left(M_{2}(x)\right)=M\left(M_{1}(x)\right) \\
& =M\left(\sum_{j=0}^{m-1} M\left(\sum_{i=0}^{n-1} x_{i j} \mathbf{1}_{A_{i}}\right) \mathbf{1}_{B_{j}}\right) \\
& =G\left(M\left(\sum_{i=0}^{n-1} x_{i 0} \mathbf{1}_{A_{i}}\right), \ldots, M\left(\sum_{i=0}^{n-1} x_{i m-1} \mathbf{1}_{A_{i}}\right)\right) \\
& =G\left(F\left(x_{00}, \ldots, x_{n-10}\right), \ldots, F\left(x_{0 m-1}, \ldots, x_{n-1 m-1}\right)\right) .
\end{aligned}
$$

Moreover, the functions $F, G$ are strictly increasing, which follows from the assumption (2.14) (cf. Remark 2.1). We could now use results on generalized bisymmetry from [2] or [9] but we will apply a slightly different method.

We will use in the sequel the following.

Definition 2.2. Let $\mathcal{F} \subset \mathcal{M}(\Omega)\left(\mathcal{M}_{+}(\Omega)\right)$. Suppose that a functional $M: \mathcal{F} \rightarrow$ $\mathbb{R}$ satisfies (2.15) for every admissible $x: \Omega \times \Omega \rightarrow \mathbb{R}$. Then we call $M$ bisymmetric.

Let us conclude the present section with the following remark.

Remark 2.4. We are going to give a representation of functionals $M$ satisfying some additional conditions. We will start with determining $M(y)$ for finite valued functions $y: \Omega \longrightarrow \mathbb{R}\left(\mathbb{R}_{+}\right)$. To determine $M(y)$ we will use the Theorem 1.2. However, this theorem works "separately" in every dimension $n \in \mathbb{N}$. The dimension is strictly connected with the partition generating $y$, i.e. such partition $\Pi=\left(A_{0}, \ldots, A_{n-1}\right) \in \mathbf{P}(\Omega)$ that $y=\sum_{i=0}^{n-1} x_{i} \mathbf{1}_{A_{i}}$. However, any given finite function may be generated by different partitions. For 
instance the function $y=\left(x_{0}, x_{1}, x_{1}\right)$ is generated by partitions $\left(A_{0}, A_{1}, A_{2}\right)$ and $\left(A_{0}, \Omega \backslash A_{0}\right)$. Therefore in the sequel we have to prove that the definition of $M(y)$ is independent of the partitions generating $y$, in particular that we may use the same generating function $\varphi$ for every partition. We will do it in the proof of Proposition 3.1 below.

\section{Results}

\subsection{Finite valued functions}

First, let us prove the following.

Proposition 3.1. Let $(\Omega, \mathcal{A}, \mu)$ be a measure space and suppose that $\mathcal{F} \subset \mathcal{M}(\Omega)$ $\left(\mathcal{M}_{+}(\Omega)\right)$ is such that $\mathbf{F}(\Omega) \subset \mathcal{F}\left(\mathbf{F}_{+}(\Omega) \subset \mathcal{F}\right)$. If $M: \mathcal{F} \rightarrow \mathbb{R}$ makes no difference between functions equal $\mu$-a.e. (2.6), is reflexive (2.7), $\mu$-strictly increasing (2.8), continuous (in the sense that $M(f)=\lim _{n \rightarrow \infty} M\left(f_{n}\right)$ for every sequence $\left(f_{n}\right)_{n \in \mathbb{N}} \in \mathcal{F}^{\mathbb{N}}$ converging a.e. to $\left.f \in \mathcal{F}\right)$ and is bisymmetric $(2.15)$ then there exist a strictly increasing and continuous function $\varphi: \mathbb{R} \rightarrow \mathbb{R}(\varphi$ : $\left.\mathbb{R}_{+} \rightarrow \mathbb{R}\right)$ and an additive function $P: \mathcal{A} \rightarrow[0,1]$ such that

$$
\begin{aligned}
& P(\Omega)=1, \\
& \bigwedge_{A \in \mathcal{A}}(P(A)>0 \Longleftrightarrow \mu(A)>0),
\end{aligned}
$$

and

$$
M(y)=\varphi^{-1}\left(\int_{\Omega}(\varphi \circ y) d P\right)
$$

for every $y \in \mathbf{F}(\Omega)\left(\mathbf{F}_{+}(\Omega)\right)$.

Proof. I. Our aim is to apply Theorem 1.2 to prove our result, with $I=\mathbb{R}$ $\left(\mathbb{R}_{+} \cdot\right)$ Let us start with a partition $\Pi=\left(A_{0}, \ldots, A_{n-1}\right) \in \mathbf{P}(\Omega)$ with $\mu\left(A_{i}\right)>$ $0, i \in\{0, \ldots, n-1\}$. Define $F_{\Pi}: \mathbb{R}^{n} \rightarrow \mathbb{R}\left(F_{\Pi}: \mathbb{R}_{+}^{n} \rightarrow \mathbb{R}\right)$ by

$$
F_{\Pi}\left(x_{0}, x_{1}, \ldots, x_{n-1}\right)=M\left(\sum_{i=0}^{n-1} x_{i} \mathbf{1}_{A_{i}}\right) \text {. }
$$

Repeating the argument from Remark 2.3 we see that $F_{\Pi}$ satisfies the bisymmetry Eq. (1.4), as well as (1.5), due to the reflexivity of $M$. Moreover, it is strictly increasing (cf. Remark 2.3) and continuous in the usual sense, as a function mapping $\mathbb{R}^{n}$ into $\mathbb{R}$. Hence by Theorem 1.2 we get that there are a strictly increasing and continuous function $\varphi_{\Pi}: \mathbb{R} \longrightarrow \mathbb{R}\left(\varphi_{\Pi}: \mathbb{R}_{+} \longrightarrow \mathbb{R}\right)$ and positive $\alpha_{\Pi, i}, i \in\{0, \ldots, n-1\}$, such that $\sum_{i=0}^{n-1} \alpha_{\Pi, i}=1$ and

$$
F_{\Pi}\left(x_{0}, x_{1}, \ldots, x_{n-1}\right)=\varphi_{\Pi}^{-1}\left(\sum_{i=0}^{n-1} \alpha_{\Pi, i} \varphi_{\Pi}\left(x_{i}\right)\right)=M\left(\sum_{i=0}^{n-1} x_{i} \mathbf{1}_{A_{i}}\right)
$$


for all $\left(x_{0}, \ldots, x_{n-1}\right) \in \mathbb{R}^{n}\left(\mathbb{R}_{+}^{n}\right)$. Let us note that (3.4) holds for all functions $y \in E_{\Pi} \subset \mathbf{F}(\Omega)\left(\mathbf{F}_{+}(\Omega)\right)$, where $E_{\Pi}$ is the linear space of functions generated by the partition $\Pi$ in the sense that

$$
y=\sum_{i=0}^{n-1} x_{i} \mathbf{1}_{A_{i}}
$$

for some (fixed) $I \ni x_{i}$ 's, $i \in\{0, \ldots, n-1\}$. In particular, if $\mu\left(A_{0}\right) \in(0,1)$ then formula (3.4) holds for any function $w_{(s, t)} \in E_{\Pi}$ defined as follows.

$$
w_{(s, t)}(p)=\left\{\begin{array}{l}
s \text { if } p \in A_{0}, \\
t \text { if } p \in \Omega \backslash A_{0} .
\end{array}\right.
$$

From (3.4) we infer that for every $s, t \in I$ the equality

$$
M\left(w_{(s, t)}\right)=\varphi_{\Pi}^{-1}\left(\alpha_{\Pi, 0} \varphi_{\Pi}(s)+\left(1-\alpha_{\Pi, 0}\right) \varphi_{\Pi}(t)\right)
$$

holds.

We are going to show that in (3.4) we may consider a function $\varphi$ instead of $\varphi_{\Pi}$, in other words we are going to show that the $\varphi_{\Pi}$ in the value of $M(y)$ is independent of the partition generating $y$ (cf. Remark 2.4). Indeed, suppose that there is a partition $\Pi^{\prime}=\left(B_{0}, \ldots, B_{m-1}\right) \in \mathbf{P}(\Omega)$ such that $\mu\left(B_{j}\right)>0, j \in$ $\{0, \ldots, m-1\}$ and

$$
\mathbf{F}(\Omega) \ni y=\sum_{i=0}^{n-1} x_{i} \mathbf{1}_{A_{i}}=\sum_{j=0}^{m-1} x_{j}^{\prime} \mathbf{1}_{B_{j}}
$$

Put

$$
F_{\Pi^{\prime}}\left(x_{0}^{\prime}, \ldots, x_{m-1}^{\prime}\right)=M\left(\sum_{j=0}^{m-1} x_{j}^{\prime} \mathbf{1}_{B_{j}}\right)=M(y) .
$$

Similarly as above we can prove that there are a strictly increasing and continuous function $\varphi_{\Pi^{\prime}}: \mathbb{R} \longrightarrow \mathbb{R}\left(\varphi_{\Pi^{\prime}}: \mathbb{R}_{+} \longrightarrow \mathbb{R}\right)$ and positive numbers $\beta_{\Pi^{\prime}, j}, j \in\{0, \ldots, m-1\}$ such that

$$
F_{\Pi^{\prime}}\left(x_{0}^{\prime}, \ldots, x_{m-1}^{\prime}\right)=\varphi_{\Pi^{\prime}}^{-1}\left(\sum_{j=0}^{m-1} \beta_{\Pi^{\prime}, j} \varphi_{\Pi^{\prime}}\left(x_{j}^{\prime}\right)\right)
$$

for all $\left(x_{0}^{\prime}, \ldots, x_{m-1}^{\prime}\right) \in I^{m}$, or for all $y \in E_{\Pi^{\prime}}$. Since

$$
F_{\Pi}\left(x_{0}, \ldots, x_{n-1}\right)=F_{\Pi^{\prime}}\left(x_{0}^{\prime}, \ldots, x_{m-1}^{\prime}\right)=M(y),
$$

taking (3.4) and (3.7) into account we obtain

$$
\varphi_{\Pi}^{-1}\left(\sum_{i=0}^{n-1} \alpha_{\Pi, i} \varphi_{\Pi}\left(x_{i}\right)\right)=\varphi_{\Pi^{\prime}}^{-1}\left(\sum_{j=0}^{m-1} \beta_{\Pi^{\prime}, j} \varphi_{\Pi^{\prime}}\left(x_{j}^{\prime}\right)\right)=M(y),
$$


for every $y \in E_{\Pi} \cap E_{\Pi^{\prime}}$. On the other hand, one also has (let us recall that $\left.\Pi \wedge \Pi^{\prime}=\left(A_{0} \cap B_{0}, \ldots, A_{i} \cap B_{j}, \ldots, A_{n-1} \cap B_{m-1}\right)\right)$

$$
y=\sum_{i=0}^{n-1} \sum_{j=0}^{m-1} c_{\Pi \wedge \Pi^{\prime}, i j} \mathbf{1}_{A_{i} \cap B_{j}}
$$

for some $c_{\Pi \wedge \Pi^{\prime}, i j}, i \in\{0, \ldots, n-1\}, j \in\{0, \ldots, m-1\}$. Taking Remark 2.2 into account, we will replace the above by a function $y^{\prime}$ equal a.e. to $y$ and given by

$$
y^{\prime}=\sum_{(i, j) \in L(m, n)} c_{\Pi \wedge \Pi^{\prime}, i j} \mathbf{1}_{C_{i j}},
$$

where $\mu\left(C_{i j}\right)>0,(i, j) \in L(m, n)$, with $L(m, n)$ defined as in Remark 2.2. Using Theorem 1.2 together with (2.6) again we get

$$
M(y)=M\left(y^{\prime}\right)=\varphi^{-1}\left(\sum_{(i, j) \in L(m, n)} \gamma_{\Pi \wedge \Pi^{\prime}, i j} \varphi\left(c_{\Pi \wedge \Pi^{\prime}, i j}\right)\right)
$$

for some strictly increasing $\varphi: \mathbb{R} \longrightarrow \mathbb{R}$ and some positive constants $\gamma_{\Pi \wedge \Pi^{\prime}, i j}$ summing up to 1 . Putting now $J:=\{j \in\{0, \ldots, m-1\}:(0, j) \in L(m, n)\} \neq \emptyset$ (the set $J$ is nonempty because $\mu\left(A_{0}\right)>0$ and $\Pi^{\prime} \in \mathbf{P}(\Omega)$ ), we see that for the function $w_{(s, t)}$ defined by (3.5) we obtain

$$
M\left(w_{(s, t)}\right)=\varphi^{-1}\left(\delta_{0} \varphi(s)+\left(1-\delta_{0}\right) \varphi(t)\right),
$$

where $\delta_{0}:=\sum_{j \in J} \gamma_{\Pi \wedge \Pi^{\prime}, 0 j}$.

Combining (3.10) with (3.6) (cf. (3.8)) and denoting $\omega=\varphi \circ \varphi_{\Pi}^{-1}$ we obtain the equation

$$
\omega\left(\alpha_{\Pi, 0} \varphi_{\Pi}(s)+\left(1-\alpha_{\Pi, 0}\right) \varphi_{\Pi}(t)\right)=\delta_{0} \varphi(s)+\left(1-\delta_{0}\right) \varphi(t),
$$

for all $s, t \in I$ or, after substituting $u:=\varphi_{\Pi}(s)$ and $v:=\varphi_{\Pi}(t)$

$$
\omega\left(\alpha_{\Pi, 0} u+\left(1-\alpha_{\Pi, 0}\right) v\right)=\delta_{0} \omega(u)+\left(1-\delta_{0}\right) \omega(v),
$$

for all $u, v \in \varphi_{\Pi}(I)=: K$, where $K$ is a real non-degenerate interval.

Applying a trick used by Daróczy and Páles [4] we see that

$$
\omega\left(\frac{u+v}{2}\right)=\frac{\omega(u)+\omega(v)}{2},
$$

for all $u, v \in K$. This yields

$$
\omega(u)=c u+d
$$

for some $c>0$ and $d \in \mathbb{R}$ and every $u \in K$. Therefore, recalling the definition of $\omega$ we get

$$
\varphi(\xi)=c \varphi_{\Pi}(\xi)+d
$$

or

$$
\varphi_{\Pi}(\xi)=c^{-1} \varphi(\xi)-d,
$$


for every $\xi \in \mathbb{R}\left(\mathbb{R}_{+}\right)$. In an analogous way we obtain

$$
\varphi_{\Pi^{\prime}}(\xi)=c^{\prime-1} \varphi(\xi)-d^{\prime},
$$

for some constants $c^{\prime}>0$ and $d^{\prime} \in \mathbb{R}$, and all $\xi \in \mathbb{R}\left(\mathbb{R}_{+}\right)$. It easily follows from (3.12) and (3.13) that (cf. (3.8))

$$
M(y)=\varphi^{-1}\left(\sum_{i=0}^{n-1} \alpha_{\Pi, i} \varphi\left(x_{i}\right)\right)=\varphi^{-1}\left(\sum_{j=0}^{m-1} \beta_{\Pi^{\prime}, j} \varphi\left(x_{j}^{\prime}\right)\right),
$$

for every $y \in \mathbf{F}(\Omega)$, independently of its representation. In other words, we get that the same $\varphi$ is enough to represent $M(y)$, for every $y \in \mathbf{F}(\Omega)\left(\mathbf{F}_{+}(\Omega)\right)$.

It remains to deal with the case $\mu\left(A_{0}\right)=1$ but then $y=c \mathbf{1}$ a.e. and thus $M(y)=c$ in view of (2.7), and it is a simple exercise to show that this value is independent of the partition generating $y$ (and moreover $M(y)=\varphi^{-1}(1 \cdot \varphi(c)$ ) for any strictly increasing and continuous function $\varphi: I \rightarrow \mathbb{R})$.

II. Suppose now that $y, z \in \mathbf{F}(\Omega)\left(\mathbf{F}_{+}(\Omega)\right)$ are two different functions. Up to now we have checked that $M(y)$ and $M(z)$ are generated by some monotone functions $\varphi$ and $\psi$, respectively. We are going to show that actually $\varphi$ and $\psi$ are conjugated. To prove it we shall use the assumed equality (2.15) to the function $x \in \mathbf{F}(\Omega \times \Omega)\left(\mathbf{F}_{+}(\Omega \times \Omega)\right)$ given by

$$
x(s, t)=\sum_{i=0}^{m-1} \sum_{j=0}^{n-1} y_{i} z_{j} \mathbf{1}_{A_{i}}(s) \mathbf{1}_{B_{j}}(t)=y(s) z(t),
$$

for all $(s, t) \in \Omega \times \Omega$, where $\left(A_{0}, \ldots, A_{n-1}\right),\left(B_{0}, \ldots, B_{m-1}\right) \in \mathbf{P}(\Omega)$ are such that

$$
y=\sum_{i=0}^{n-1} y_{i} \mathbf{1}_{A_{i}} \text { and } z=\sum_{j=0}^{m-1} z_{j} \mathbf{1}_{B_{j}} .
$$

In view of (2.12) and (2.13) we obtain

$$
M_{1}(x)=\sum_{j=0}^{m-1} M\left(z_{j} \sum_{i=0}^{n-1} y_{i} \mathbf{1}_{A_{i}}\right) \mathbf{1}_{B_{j}}=\sum_{j=0}^{m-1} M\left(z_{j} y\right) \mathbf{1}_{B_{j}},
$$

and

$$
M_{2}(x)=\sum_{i=0}^{n-1} M\left(y_{i} \sum_{j=0}^{m-1} z_{j} \mathbf{1}_{B_{j}}\right) \mathbf{1}_{A_{i}}=\sum_{i=0}^{n-1} M\left(y_{i} z\right) \mathbf{1}_{A_{i}} .
$$

Let us take $y=y_{0} \mathbf{1}_{A_{0}}+y_{1} \mathbf{1}_{\Omega \backslash A_{0}}$ and $z=z_{0} \mathbf{1}_{B_{0}}+z_{1} \mathbf{1}_{\Omega \backslash B_{0}}$ (it is rather obvious that it is enough to restrict our attention to this special case). Then 
(2.15) becomes

$$
\begin{aligned}
\psi^{-1} & \left(\beta_{0} \psi\left(\varphi^{-1}\left(\alpha_{0} \varphi\left(y_{0} z_{0}\right)+\left(1-\alpha_{0}\right) \varphi\left(y_{1} z_{0}\right)\right)\right)\right. \\
& \left.+\left(1-\beta_{0}\right) \psi\left(\varphi^{-1}\left(\alpha_{0} \varphi\left(y_{0} z_{1}\right)+\left(1-\alpha_{0}\right) \varphi\left(y_{1} z_{1}\right)\right)\right)\right) \\
& =\varphi^{-1}\left(\alpha_{0} \varphi\left(\psi^{-1}\left(\beta_{0} \psi\left(y_{0} z_{0}\right)+\left(1-\beta_{0}\right) \psi\left(y_{1} z_{0}\right)\right)\right)\right. \\
& \left.+\left(1-\alpha_{0}\right) \varphi\left(\psi^{-1}\left(\beta_{0} \psi\left(y_{0} z_{1}\right)+\left(1-\beta_{0}\right) \psi\left(y_{1} z_{1}\right)\right)\right)\right) .
\end{aligned}
$$

Substituting $s=y_{0} z_{0}, t=y_{1} z_{0}, u=y_{0} z_{1}$, and $v=y_{1} z_{1}$ above we get

$$
\begin{aligned}
\psi^{-1} & \left(\beta_{0} \psi\left(\varphi^{-1}\left(\alpha_{0} \varphi(s)+\left(1-\alpha_{0}\right) \varphi(t)\right)\right)\right. \\
& \left.+\left(1-\beta_{0}\right) \psi\left(\varphi^{-1}\left(\alpha_{0} \varphi(u)+\left(1-\alpha_{0}\right) \varphi(v)\right)\right)\right) \\
& =\varphi^{-1}\left(\alpha_{0} \varphi\left(\psi^{-1}\left(\beta_{0} \psi(s)+\left(1-\beta_{0}\right) \psi(t)\right)\right)\right. \\
& \left.+\left(1-\alpha_{0}\right) \varphi\left(\psi^{-1}\left(\beta_{0} \psi(u)+\left(1-\beta_{0}\right) \psi(v)\right)\right)\right) .
\end{aligned}
$$

However, this is an equation that was treated by Leonetti et al. [8] in a slightly more general situation (cf. also Theorem 2 in [6]). They showed (cf. Lemma 3 in [8]) that there exist real constants $a, b$ with $a \neq 0$ such that

$$
\varphi=a \psi+b
$$

A straightforward calculation shows that both $\varphi$ and $\psi$ generate the same $M$, more exactly we have $M(y)=\varphi^{-1}\left(\sum_{i=0}^{n-1} \alpha_{i} \varphi\left(y_{i}\right)\right)$ and $M(z)=\varphi^{-1}$ $\left(\sum_{j=0}^{m-1} \beta_{j} \varphi\left(z_{j}\right)\right)$.

III. Let us pick a set $A \in \mathcal{A}$ and consider the partition $\Pi=(A, \Omega \backslash A) \in \mathbf{P}(\Omega)$. If $y \in \mathbf{F}(\Omega)\left(\mathbf{F}_{+}(\Omega)\right)$ is given by $y=y_{0} \mathbf{1}_{A}+y_{1} \mathbf{1}_{\Omega \backslash A}$ and $\mu(A) \cdot \mu(\Omega \backslash A)>0$ then, as we have shown above, there exist a strictly monotone and continuous function $\varphi: \mathbb{R} \rightarrow \mathbb{R}\left(\mathbb{R}_{+} \rightarrow \mathbb{R}\right)$ and a number $\alpha_{\Pi} \in(0,1)$ such that $M(y)=$ $\varphi^{-1}\left(\alpha_{\Pi} \varphi\left(y_{0}\right)+\left(1-\alpha_{\Pi}\right) \varphi\left(y_{1}\right)\right)$. Now, define (cf. (3.4))

$$
P(A)=\left\{\begin{aligned}
0 & \text { if } \mu(A)=0 \\
\alpha_{\Pi} & \text { if } \mu(A)>0 \\
1 & \text { if } \mu(\Omega \backslash A)=0 .
\end{aligned}\right.
$$

We have to check that

1. $P$ is well defined, i.e. the definition of $P(A)$ does not depend on the partition,

2. $P$ is additive,

3. (3.2) holds.

Ad 1. Suppose that there is another partition $\Pi^{\prime}=\left(A, B_{1}, \ldots, B_{m-1}\right)$ and $y$ is given by $y=y_{0} \mathbf{1}_{A}+y_{1} \mathbf{1}_{\Omega \backslash A}=y_{0} \mathbf{1}_{A}+\sum_{j=1}^{m-1} y_{j}^{\prime} \mathbf{1}_{B_{j}}$. We have shown above that then $y$ is also given by (3.9), which in the present case takes the form 
(put $J=\left\{j \in\{1, \ldots, m-1\}: \mu\left(A \cap B_{j}\right)>0\right\}, J^{\prime}=\{j \in\{1, \ldots, m-1\}$ : $\left.\left.\mu\left((\Omega \backslash A) \cap B_{j}\right)>0\right\}\right)$

$$
y=c_{\Pi \wedge \Pi \Pi^{\prime}, 00} \mathbf{1}_{A}+\sum_{j \in J} c_{\Pi \wedge \Pi \Pi^{\prime}, 0 j} \mathbf{1}_{A \cap B_{j}}+\sum_{j \in J^{\prime}} c_{\Pi \wedge \wedge \Pi^{\prime}, 1 j} \mathbf{1}_{(\Omega \backslash A) \cap B_{j}} .
$$

Put $y_{0}=c_{\Pi \wedge \Pi^{\prime}, 0 j}, j \in\{0\} \cup J$ and $y_{1}=c_{\Pi \wedge \Pi^{\prime}, 1 j}, j \in J^{\prime}$. Put $\gamma_{0}:=\sum_{j \in\{0\} \cup J}$ $\gamma_{\Pi \wedge \wedge \Pi^{\prime}, 0 j}$. Then we get (cf. (3.14))

$$
\begin{aligned}
F\left(y_{0}, y_{1}, \ldots, y_{1}\right) & =\varphi^{-1}\left(\alpha_{\Pi} \varphi\left(y_{0}\right)+\left(1-\alpha_{\Pi}\right) \varphi\left(y_{1}\right)\right) \\
& =M\left(y_{0} \mathbf{1}_{A}+y_{1} \mathbf{1}_{\Omega \backslash A}\right)=\varphi^{-1}\left(\gamma_{0} \varphi\left(y_{0}\right)+\left(1-\gamma_{0}\right) \varphi\left(y_{1}\right)\right) .
\end{aligned}
$$

It follows from (3.15) that

$$
\left(\alpha_{\Pi}-\gamma_{0}\right)\left(\varphi\left(y_{0}\right)-\varphi\left(y_{1}\right)\right)=0
$$

for every $y_{0}, y_{1} \in \mathbb{R}\left(\mathbb{R}_{+}\right)$. Obviously then $\alpha_{\Pi}=\gamma_{0}$ and the correctness of the definition of $P$ is proved. In particular, we get (3.1).

Ad 2. As to the additivity of $P$, consider the following two partitions of $\Omega$ :

$$
\left(A_{0}, A_{1}, \Omega \backslash\left(A_{0} \cup A_{1}\right)\right)
$$

and

$$
\left(A_{0} \cup A_{1}, \Omega \backslash\left(A_{0} \cup A_{1}\right)\right)
$$

for some disjoint sets $A_{0}, A_{1} \in \mathcal{A}$. We may assume w.l.o.g. that $\mu\left(A_{0}\right) \mu\left(A_{1}\right) \mu$ $\left(\Omega \backslash\left(A_{0} \cup A_{1}\right)\right)>0$. Then for every $s, t \in \mathbb{R}\left(\mathbb{R}_{+}\right)$we have (cf. (2.16) or (2.17))

$$
M\left(s \mathbf{1}_{A_{0}}+s \mathbf{1}_{A_{1}}+t \mathbf{1}_{\Omega \backslash\left(A_{0} \cup A_{1}\right)}\right)=M\left(s \mathbf{1}_{A_{0} \cup A_{1}}+t \mathbf{1}_{\Omega \backslash\left(A_{0} \cup A_{1}\right)}\right),
$$

which yields (cf. (3.14) applied to the left-hand side)

$$
\varphi^{-1}\left(\alpha_{0} \varphi(s)+\alpha_{1} \varphi(s)+\alpha_{2} \varphi(t)\right)=\varphi^{-1}\left(\left(\alpha_{0}+\alpha_{1}\right) \varphi(s)+\alpha_{2} \varphi(t)\right),
$$

for all $s, t \in \mathbb{R}\left(\mathbb{R}_{+}\right)$. On the other hand we get

$$
M\left(s \mathbf{1}_{A_{0} \cup A_{1}}+t \mathbf{1}_{\Omega \backslash\left(A_{0} \cup A_{1}\right)}\right)=\varphi^{-1}\left(\beta_{0} \varphi(s)+\beta_{2} \varphi(t)\right)
$$

for some $\beta_{0}, \beta_{1}$ and all $s, t \in \mathbb{R}\left(\mathbb{R}_{+}\right)$. From (3.17), (3.17) and (3.18), and the properties of $\varphi$ we get

$$
\alpha_{0}+\alpha_{1}=\beta_{0} \text { and } \alpha_{2}=\beta_{2},
$$

which means in particular that

$$
P\left(A_{0} \cup A_{1}\right)=P\left(A_{0}\right)+P\left(A_{1}\right),
$$

and yields the required additivity of $P$.

Ad 3. Now we proceed to prove the equivalence (3.2). Suppose that $A \in \mathcal{A}$ is such that $\mu(A) \mu(\Omega \backslash A)>0$. Take $s<t$ and put $x=s \mathbf{1}_{A}+0 \mathbf{1}_{\Omega \backslash A}$ and 
$y=t \mathbf{1}_{A}+0 \mathbf{1}_{\Omega \backslash A}$. Since $M$ is $\mu$-strictly increasing, we have $M(x)<M(y)$, whence (cf. (2.16)) $F(s, 0)<F(t, 0)$, or (cf. (3.14))

$$
\varphi^{-1}(P(A) \varphi(s))<\varphi^{-1}(P(A) \varphi(t)) .
$$

It follows that $P(A)>0$.

To see that the converse also holds, suppose that for some $A \in \mathcal{A}$ we have $P(A)>0$ and $\mu(A)=0$. Then $\mu(\Omega \backslash A)>0$ and, as it follows from the first part of the present argument, $P(\Omega \backslash A)>0$. Let us take $s<t, u, v \in \mathbb{R}\left(\mathbb{R}_{+}\right)$and define two functions $f, g: \Omega \longrightarrow \mathbb{R}$ by $f=u \mathbf{1}_{A}+s \mathbf{1}_{\Omega \backslash A}$ and $g=v \mathbf{1}_{A}+t \mathbf{1}_{\Omega \backslash A}$. Then $f<g$ in the sense of (2.1). Thus $M(f)<M(g)$ by monotonicity which implies

$$
P(A) \varphi(u)+(1-P(A)) \varphi(s)<P(A) \varphi(v)+(1-P(A)) \varphi(t),
$$

whence it follows that

$$
\varphi(v)-\varphi(u)>\frac{P(\Omega \backslash A)}{P(A)}[\varphi(t)-\varphi(s)] .
$$

Taking $v<u$ and letting $s \longrightarrow t$ in (3.19) we get

$$
0>\varphi(v)-\varphi(u) \geq 0
$$

a contradiction.

IV. The formula (3.3) follows naturally. Indeed, take a $y \in \mathbf{F}(\Omega)\left(\mathbf{F}_{+}(\Omega)\right.$ ), and suppose that there exist a partition $\Pi=\left(A_{0}, \ldots, A_{n-1}\right)\left(\right.$ with $\mu\left(A_{i}\right)>0, i \in$ $\{0, \ldots, n-1\})$ such that

$$
y=\sum_{i=0}^{n-1} y_{i} \mathbf{1}_{A_{i}}
$$

for some $y_{i} \in \mathbb{R}\left(\mathbb{R}_{+}\right)$. Then there exist a strictly monotone function $\varphi$ and positive numbers $\alpha_{i}, i \in\{0, \ldots, n-1\}$ such that $\sum_{i=0}^{n-1} \alpha_{i}=1$ and

$M(y)=\varphi^{-1}\left(\sum_{i=0}^{n-1} \alpha_{i} \varphi\left(y_{i}\right)\right)=\varphi^{-1}\left(\sum_{i=0}^{n-1} P\left(A_{i}\right) \varphi\left(y_{i}\right)\right)=\varphi^{-1}\left(\int_{\Omega} f \circ y d P\right)$.

\subsection{Arbitrary case}

We have proved that a continuous, bisymmetric and reflexive functional $M$ which satisfies (2.15) is given by (3.3) for any $y \in \mathbf{F}(\Omega)\left(\mathbf{F}_{+}(\Omega)\right)$. If the set $\mathbf{F}(\Omega)\left(\mathbf{F}_{+}(\Omega)\right)$ is dense in a function family $\mathcal{F}$ (or at least any element of $\mathcal{F}$ can be approximated by a sequence from $\left.\mathbf{F}(\Omega)\left(\mathbf{F}_{+}(\Omega)\right)\right)$ then sometimes, due to the continuity of $M, \varphi$ and $\varphi^{-1}$ we may pass with lim under the sign of $\int$, and we get formula (3.3) for any $y \in X$.

Below we present two such cases: the space of bounded functions or the space of integrable functions. 
Corollary 3.1. Let $\mu: 2^{\Omega} \rightarrow[0, \infty]$ be an additive, nontrivial function. Then $M: B(\Omega, \mathbb{R}) \rightarrow \mathbb{R}$ is reflexive, $\mu$-strictly increasing, continuous and bisymmetric if, and only if, there exist a strictly increasing and continuous function $\varphi: \mathbb{R} \rightarrow \mathbb{R}$ and an additive function $P: 2^{\Omega} \rightarrow[0,1]$ such that (3.1), (3.2) are satisfied and (3.3) holds for every $y \in B(\Omega, \mathbb{R})$.

Proof. In the space $\mathbf{F}(\Omega)$ we have defined the functional $M$ by the formula

$$
M(x)=\varphi^{-1}\left(\int_{\Omega}(\varphi \circ x) d P\right) .
$$

Now, this functional can be uniquely extended to a continuous functional onto the whole $B(\Omega, \mathbb{R})$ because $\mathbf{F}(\Omega)$ is a dense subspace of $B(\Omega, \mathbb{R})$. Indeed, for every $x \in B(\Omega, \mathbb{R})$ there exists a sequence $\left(x_{n}\right)_{n \in \mathbb{N}} \in \mathbf{F}(\Omega)^{\mathbb{N}}$, which converges uniformly to $x$. The sequence $\left(\varphi \circ x_{n}\right)_{n \in \mathbb{N}}$ is then uniformly convergent to $\varphi \circ x$, due to the uniform continuity of $\varphi$ in any compact subset of $\mathbb{R}$. Thus we get (considering the finiteness of $P(\Omega)$ and continuity of $\varphi^{-1}$ )

$$
\begin{aligned}
\lim _{n \rightarrow \infty} & M\left(x_{n}\right)=\lim _{n \rightarrow \infty} \varphi^{-1}\left(\int_{\Omega}\left(\varphi \circ x_{n}\right) d P\right) \\
& =\varphi^{-1}\left(\lim _{n \rightarrow \infty} \int_{\Omega}\left(\varphi \circ x_{n}\right) d P\right)=\varphi^{-1}\left(\int_{\Omega} \lim _{n \rightarrow \infty}\left(\varphi \circ x_{n}\right) d P\right) \\
& =\varphi^{-1}\left(\int_{\Omega}(\varphi \circ x) d P\right)=M(x),
\end{aligned}
$$

which was to be proved. It remains to show that (3.2) holds (with $\mathcal{A}=2^{\Omega}$ ) but we have already proved it while showing Proposition 3.1.

Corollary 3.2. Let $(\Omega, \mathcal{A}, \mu)$ be a measure space with a finite $\mu$. Let $M: L^{1}(\Omega, \mathbb{R})$ $\rightarrow \mathbb{R}$ be reflexive, $\mu$-strictly increasing, continuous and bisymmetric. Then $M$ is given by (3.3) for every $y \in \mathbf{F}(\Omega)$ with some increasing and continuous $\varphi: \mathbb{R} \rightarrow \mathbb{R}$ and additive $P: \mathcal{A} \rightarrow[0,1]$. If, additionally, we assume that $\varphi$ is bounded then $M$ is given by (3.3) for every $y \in L^{1}(\Omega, \mathbb{R})$, and $P$ is a probability measure equivalent to $\mu$.

Proof. An additional assumption on $\varphi$ is needed because we want the composition $\varphi \circ y$ to be in $L^{1}(\Omega, \mathbb{R})$.

We know that $M(y)$ is given by (3.3) for every $y \in \mathbf{F}(\Omega)$ : it follows from Proposition 3.1. But $\mathbf{F}(\Omega)$ is a dense subset of $L^{1}(\Omega, \mathbb{R})$ and therefore for each $y \in L^{1}(\Omega, \mathbb{R})$ there exists a sequence $\left(y_{n}\right)_{n \in \mathbb{N}} \in \mathbf{F}(\Omega)$, converging to $y$ in the norm of $L^{1}$. Using the continuity of $M$ and the fact that $\varphi$ is a homeomorphism, we get henceforth

$$
\begin{aligned}
M(y) & =M\left(\lim _{n \rightarrow \infty} y_{n}\right)=\lim _{n \rightarrow \infty} M\left(y_{n}\right) \\
& =\lim _{n \rightarrow \infty} \varphi^{-1}\left(\int_{\Omega} \varphi \circ y_{n} d P\right)=\varphi^{-1}\left(\int_{\Omega} \varphi \circ y d P\right) .
\end{aligned}
$$


If $\mu$ is a measure, then we obtain the $\sigma$-additivity of $P$ in the following way. Suppose that $A=\bigcup_{n=1}^{\infty} A_{n}$ where $A_{n} \in \mathcal{A}$ are pairwise disjoint. Fix an $N \in \mathbb{N}$ and consider the following finite valued function $z_{N}=s \mathbf{1}_{\bigcup_{n=1}^{N} A_{n}}+t \mathbf{1}_{\Omega \backslash \bigcup_{n=1}^{N} A_{n}}$. Then

$$
\begin{aligned}
M\left(z_{N}\right) & =\varphi^{-1}\left(P\left(\bigcup_{n=1}^{N} A_{n}\right) \varphi(s)+P\left(\Omega \backslash \bigcup_{n=1}^{N} A_{n}\right) \varphi(t)\right) \\
& =\varphi^{-1}\left(\sum_{n=1}^{N} P\left(A_{n}\right) \varphi(s)+\left(1-\sum_{n=1}^{N} P\left(A_{n}\right)\right) \varphi(t)\right),
\end{aligned}
$$

and therefore

$$
\varphi\left(M\left(z_{N}\right)\right)=\left(\sum_{n=1}^{N} P\left(A_{n}\right)\right) \varphi(s)+\left(1-\sum_{n=1}^{N} P\left(A_{n}\right)\right) \varphi(t) .
$$

Since $\lim _{N \rightarrow \infty} z_{N}=s \mathbf{1}_{A}+t \mathbf{1}_{\Omega \backslash A}$ pointwise (but also in $L^{1}$-norm) we have by the continuity of $\varphi$ and $M$ that

$$
\lim _{N \rightarrow \infty} \varphi\left(M\left(z_{N}\right)\right)=P(A) \varphi(s)+(1-P(A)) \varphi(t)
$$

whence

$$
\left(\lim _{N \rightarrow \infty} \sum_{n=1}^{N} P\left(A_{n}\right)-P(A)\right)(\varphi(s)-\varphi(t))=0
$$

for every $s, t \in \mathbb{R}$ follows. Hence

$$
\sum_{n=1}^{\infty} P\left(A_{n}\right)=P(A)
$$

Without any assumption on $\varphi$ we get the following result.

Corollary 3.3. Let $(\Omega, \mathcal{A}, \mu)$ be a measure space with a finite $\mu$. Let $\mathcal{F}$ consist of all $\mathcal{A}$-measurable, bounded and non-negative functions. Then $M: \mathcal{F} \rightarrow \mathbb{R}$ is reflexive, $\mu$-strictly increasing, continuous and bisymmetric if, and only if, $M$ is given by (3.3) for every $y \in \mathcal{F}$ with some increasing and continuous $\varphi: \mathbb{R} \rightarrow \mathbb{R}$ and $P: \mathcal{A} \rightarrow[0,1]$ which is a probability measure equivalent to $\mu$.

\subsection{Final remarks}

At the 53rd International Symposium on Functional Equations J. Chudziak (cf. [3]) called our attention to the fact that there is a connection between the above results and utility theory. Namely, if $u$ is an arbitrary utility function (i.e. continuous and strictly increasing), we say that $\lambda \in \mathbb{R}$ is a certainty equivalent of a gamble $X \in \chi_{+}$iff

$$
u(\lambda)=\mathbb{E}(u(X))
$$


whence

$$
\lambda=u^{-1}[\mathbb{E}(u(X))] .
$$

Here the symbol $\chi_{+}$denotes all the non-negative and bounded random variables. Let us note that the assumption of boundedness, which guarantees the correctness of the definition (3.20) could be replaced by the requirement of concavity for $u$. Indeed then the Jensen inequality is implied, and one has

$$
0 \leq \int_{\Omega} u \circ y d P \leq u\left(\int_{\Omega} y d P\right)<\infty
$$

for all $y \in L^{1}\left(\Omega, \mathbb{R}_{+}\right)$.

The functional $M: \chi_{+} \longrightarrow \mathbb{R}$ given by

$$
M(X):=u^{-1}[\mathbb{E}(u(X))]=u^{-1}\left(\int_{\Omega} u(X) d P\right)
$$

is called equivalence functional.

Thus we can conclude our present considerations by the following.

Corollary 3.4. Let $(\Omega, \mathcal{A}, \mu)$ be a probability space. Let $\chi_{+}$consist of all $\mathcal{A}$-measurable, bounded and non-negative functions. Then $M: \chi_{+} \rightarrow \mathbb{R}$ is reflexive, $\mu$-strictly increasing, continuous and bisymmetric if, and only if, $M$ is given by (3.21) for every $X \in \chi_{+}$, where $u: \mathbb{R}_{+} \rightarrow \mathbb{R}$ is a utility function and $\mathbb{E}$ is the expectation taken with respect to some probability measure $P$, equivalent to $\mu$.

We also have

Corollary 3.5. Let $(\Omega, \mathcal{A}, \mu)$ be a probability space. Let $M: L^{1}\left(\Omega, \mathbb{R}_{+}\right) \longrightarrow \mathbb{R}$ be reflexive, $\mu$-strictly increasing, continuous and bisymmetric. Then, according to Proposition 3.1, there exist a probability measure $P$ equivalent to $\mu$ and $a$ continuous and strictly increasing function $u: \mathbb{R}_{+} \rightarrow \mathbb{R}$ such that $M$ is given by (3.21) for all $X \in \mathbf{F}_{+}(\Omega)$. If, additionally, $u$ is concave and non-negative then $M$ is given by (3.21) for all $X \in L^{1}\left(\Omega, \mathbb{R}_{+}\right)$.

\section{Acknowledgements}

We are grateful to a referee whose remarks and questions have helped us in making the present work better, easier to read and to understand.

Open Access. This article is licensed under a Creative Commons Attribution 4.0 International License, which permits use, sharing, adaptation, distribution and reproduction in any medium or format, as long as you give appropriate credit to the original author(s) and the source, provide a link to the Creative Commons licence, and indicate if changes were made. The images or other third party material in this article are included in the article's Creative Commons licence, unless indicated otherwise in a credit line to the material. If material is not included in the article's Creative Commons licence and your intended use is not permitted by statutory regulation or exceeds the permitted use, you will need to obtain 
permission directly from the copyright holder. To view a copy of this licence, visit http:// creativecommons.org/licenses/by/4.0/.

Publisher's Note Springer Nature remains neutral with regard to jurisdictional claims in published maps and institutional affiliations.

\section{References}

[1] Aczél, J.: Lectures on Functional Equations and their Applications. Academic Press, New York (1966)

[2] Aczél, J., Maksa, Gy., Taylor, M.: Equations of generalized bisymmetry and of consistent aggregation: weakly surjective solutions which may be discontinuous at places. J. Math. Anal. Appl. 214, 22-35 (1997)

[3] Chudziak, J.: Personal communication. In: 53rd International Symposium on Functional Equations, Krynica, Poland (2015)

[4] Convexity with given weight sequences: Daróczy, Z., Páles, Zs. Stochastica 11, 5-12 (1987)

[5] de Finetti, B.: Sul concetto di media. Giornale dell'Istituto Italiano degli Attuari 2, 369-396 (1931)

[6] Głazowska, D., Leonetti, P., Matkowski, J., Tringali, S.: Commutativity of integral quasiarithmetic means on measure spaces. Acta Math. Hungar. 153(2), 350-355 (2017)

[7] Hardy, G.H., Littlewood, J.E., Pólya, G.: Inequalities. Cambridge University Press, Cambridge (1952)

[8] Leonetti, P., Matkowski, J., Tringali, S.: On the commutation of generalized means on probability spaces. Indagationes Math. 27, 945-953 (2016)

[9] Maksa, Gy.: Solution of generalized bisymmetry type equations without surjectivity assumptions. Aequationes Math. 57, 50-74 (1999)

[10] Maksa, Gy.: Quasisums and generalized associativity. Aequationes Math. 69, 6-27 (2005)

[11] Münnich, A., Maksa, Gy., Mokken, R.J.: n-Variable bisection. J. Math. Psychol. 44, $569-581(2000)$

[12] Volkmann, P.: Oral remark during the discussion at the Institute of Mathematics of the University of Silesia, unpublished (2009)

Maciej Sablik

Institute of Mathematics

University of Silesia

Bankowa 14

40007 Katowice

Poland

e-mail: maciej.sablik@us.edu.pl

Received: March 30, 2019

Revised: May 7, 2020 